\title{
Time-series spectroscopy and photometry of the pulsating subdwarf B star PG 1219+534 (KY UMa) ${ }^{\star}$
}

\author{
M. D. Reed ${ }^{1, \star \star}$, J. R. Eggen ${ }^{1}$, S. L. Harms ${ }^{1}$, J. H. Telting ${ }^{2}$, R. H. Østensen ${ }^{3}$, S. J. O’ Toole ${ }^{4}$, D. M. Terndrup ${ }^{5}$, \\ A.-Y. Zhou ${ }^{1,6}$, R. L. Kienenberger ${ }^{1}$, and U. Heber ${ }^{7}$ \\ 1 Department of Physics, Astronomy, \& Materials Science, Missouri State University, 901 S. National, Springfield, MO 65897, USA \\ e-mail: mreed@sdbv.missouristate.edu; [joe,trillianlala, aiyingzhou,r.vanwey]@gmail.com \\ 2 Nordic Optical Telescope, Apartado 474, 38700 Santa Cruz de La Palma, Spain \\ e-mail: jht@not.iac.es \\ ${ }^{3}$ Institute of Astronomy, Catholic University of Leuven, Celestijnenlaan 200B, 3001 Leuven, Belgium \\ e-mail: roy@ster.kuleuven.ac.be \\ 4 Anglo-Australian Observatory, PO Box 296, Epping NSW 1710, Australia \\ e-mail: otoole@aao.gov.au \\ 5 Department of Astronomy, The Ohio State University, Columbus, OH 43210, USA \\ e-mail: terndrup@astronomy . ohio-state.edu \\ 6 National Astronomical Observatories of the Chinese Academy of Sciences, Beijing 100012, PR China \\ 7 Dr. Remeis-Sternwarte Bamberg, Universität Erlangen-Nürnberg, Sternwartstr 7, 96049 Bamberg, Germany \\ e-mail: Ulrich.Heber@sternwarte.uni-erlangen.de
}

Received 9 August 2008 / Accepted 26 October 2008

\section{ABSTRACT}

\begin{abstract}
Aims. We present observations and analysis of time-series spectroscopy and photometry of the pulsating subdwarf B star PG 1219+534 (KY UMa). Subdwarf B stars are blue horizontal branch stars that have shed most of their hydrogen envelopes. Pulsating subdwarf B stars allow a probe into this interesting phase of evolution.

Methods. Low-resolution spectra were obtained at the Nordic Optical Telescope and Kitt Peak National Observatory, and photometric observations were obtained at MDM and Baker observatories in 2006. We extracted radial velocity and equivalent width variations from several Balmer and He I lines in individual spectra. The pulsation frequencies were separated via phase binning to detect lineprofile variations in Balmer and helium lines, which were subsequently matched to atmospheric models to infer effective temperature and gravity changes throughout the pulsation cycle.

Results. From the photometry we recovered the four previously observed frequencies and detected a new fifth frequency. From the spectra we directly measured radial velocity and equivalent width variations for the four main frequencies and from atmospheric models. We successfully inferred temperature and gravity changes for these four frequencies. We compared amplitude ratios and phase differences of these quantities and searched for outliers that could be identified as high-degree modes. These are the first such measurements for a "normal" amplitude pulsating subdwarf B star, indicating that spectroscopic studies can benefit the majority of pulsating subdwarf B stars.
\end{abstract}

Key words. techniques: radial velocities - stars: subdwarfs - stars: variables: general - techniques: spectroscopic stars: individual: PG 1219+534

\section{Introduction}

Subdwarf B (sdB) stars are the field counterparts to extended horizontal branch stars observed in globular clusters. Their masses are about $0.5 M_{\odot}$ with thin $\left(<10^{-2} M_{\odot}\right)$, inert hydrogen envelopes and temperatures from 22000 to $40000 \mathrm{~K}$ (Heber et al. 1984; Saffer et al. 1994), making them very blue. Their origin remains a mystery, because mass loss on the first-ascent giant branch must remove all but $<10^{-2} M_{\odot}$ of the hydrogen envelope, yet produce a core of the same mass (to within a few percent) every time (D'Cruz et al. 1996; Han et al. 2002, 2003). Pulsating

\footnotetext{
* Based on observations from the Nordic Optical Telescope, Kitt Peak National Observatory, and MDM Observatory.

$\star \star$ Visiting Astronomer, Kitt Peak National Observatory, National Optical Astronomy Observatory, which is operated by the Association of Universities for Research in Astronomy (AURA) under cooperative agreement with the National Science Foundation.
}

sdB stars come in two varieties. The short-period (90 to $600 \mathrm{~s}$ ) pulsators were discovered in 1997 (Kilkenny et al. 1997) and were named EC 14026 stars after that prototype. Their official designation is V361 Hya stars, the General Catalog of Variables Stars lists them as RPHS (Kazarovets et al. 2003), but they are typically referred to as sdBV stars. Their usual amplitudes are near $1 \%$ of their mean brightness and stars have been detected with as few as one (Reed et al. 2006, HS 1824+5745) or as many as 55 (Kilkenny et al. 1999, PG 1605+072) pulsation frequencies (Reed et al. 2007a). Long-period (45 min to $2 \mathrm{~h}$ ) pulsators were discovered in 2003 (Green et al. 2003; Reed et al. 2004a) and are officially designated as V1093 Her stars but commonly named PG 1716 stars after that prototype, PG 1716+426. Their amplitudes are typically $<0.1 \%$ and they tend to be multimode pulsators. For this work, our interest is the sdBV class of pulsators as their periods are short, so many pulsation cycles can be observed during each run from a single site. The short periods 
Table 1. 2006 Photometric observations of PG 1219 simultaneous or near to the spectroscopic observations.

\begin{tabular}{lccccc}
\hline \hline $\begin{array}{l}\text { Run } \\
\text { Name }\end{array}$ & $\begin{array}{c}\text { Date } \\
\text { (UT) }\end{array}$ & $\begin{array}{c}\text { Start time } \\
\text { (UT) }\end{array}$ & $\begin{array}{c}\text { Length } \\
\text { (h) }\end{array}$ & $\begin{array}{c}\text { Number of } \\
\text { images }\end{array}$ & Observat. \\
\hline mdm040806 & 8 Apr. & $3: 10: 30$ & 9.3 & 3900 & MDM \\
mdm040906 & 9 Apr. & $3: 21: 30$ & 4.4 & 1916 & MDM \\
mdm041006 & 10 Apr. & $2: 24: 52$ & 2.8 & 521 & MDM \\
mdm041106 & 11 Apr. & $4: 23: 30$ & 3.5 & 1132 & MDM \\
bak041806 & 18 Apr. & $5: 09: 30$ & 4.9 & 1583 & BO \\
bak041906 & 19 Apr. & $2: 23: 30$ & 7.7 & 2056 & BO \\
bak042006 & 20 Apr. & $2: 20: 00$ & 6.6 & 1815 & BO \\
bak042206 & 22 Apr. & $2: 25: 00$ & 7.4 & 2030 & BO \\
\hline
\end{tabular}

also produce spectroscopic variations (radial velocity, equivalent width, effective temperature and gravity) at an observable level for intermediate resolution spectrographs and $4 \mathrm{~m}$ class telescopes.

Pulsating sdB stars potentially allow the opportunity to discern their interior structure using asteroseismology to obtain estimates of total mass, luminosity, envelope mass, radiative levitation, gravitational diffusion, and helium fusion cross sections. To do so, the pulsation modes must first be identified with their associated spherical harmonics. In 1999, O'Toole et al. (2000, 2002, 2003) began applying time-series spectroscopic techniques to attempt mode identification in sdBV stars. Whereas photometry measures brightness changes largely caused by temperature variations, spectroscopy can reveal information regarding the pulsation velocities and separate the temperature and gravity components. An excellent example of these techniques was shown in Telting \& Østensen (2004) where they determined that the pulsational degree of the highestamplitude frequency of PG $1325+101$ (QQ Vir) was consistent with $\ell=0$. Other sdBV stars studied using time-series spectroscopy include PB 8783 (EO Cet) and KPD 2109+4401 (V2203 Cyg) (Jefferey \& Pollacco 2000), PG 1605+072 (V338 Ser) (O'Toole et al. 2000, 2002, 2003, 2005; Woolf et al. 2002), and Balloon 090100001 (Telting \& Østensen 2006) using low-resolution spectroscopy on intermediate-sized telescopes. PG 1219+534, PG 1605+072, and PG 1613+426 were also observed using FUSE (Kuassivi et al. 2005). Only for the high-amplitude pulsators (PG 1605+072, PG 1325+101, and Balloon 090100001) were pulsations detected. However, such pulsators provide their own problems in that the high-amplitude pulsations affect the other frequencies and therefore require considerable prewhitening (variation removal); e.g. Telting \& Østensen (2006); Tillich et al. (2007). Unfortunately, because of insufficient signal, temporal resolution, or run length, none of these studies have provided conclusive mode identifications which would be useful for constraining models.

This paper reports our time-series spectroscopic and photometric observations of PG 1219+534 (also KY UMa but hereafter PG 1219). PG 1219 was discovered to be a pulsator by Koen et al. (1999) who detected four independent frequencies. The spacings of the frequencies are such that the entire pulsation spectrum can be resolved in just a few hours, yet the frequency density is too large for the pulsations to be of the same modal degree $\ell$. A photometry-based mode identification has been published which attributes the four frequencies to three differing modal degrees (Charpinet et al. 2005) which can be used as a guide for our mode identifications. While the pulsation amplitudes are low (2-8 mma in photometry), they should not interfere with each other, eliminating the need to prewhiten some frequencies to reveal others. This makes PG 1219 an excellent target for mode identification studies. Our photometric observations of PG 1219 began in 2003 as part of our effort to resolve the pulsation spectra (Fourier transform; FT) of sdBV stars (Reed et al. 2004b, 2006, 2007a,b; Zhou et al. 2006); Harms et al. (2006) reported the results of 2003-2005 photometric observations of PG 1219. During that time, the pulsation frequencies remained consistent with those of Koen et al. (1999) and occasionally a new, low-amplitude frequency would appear for one or two nights.

In Sect. 2 we describe our simultaneous spectroscopic and photometric observations during 2006 and examine the frequency content in Sect. 3. In Sect. 4 we discuss the results of our observations and in Sect. 5 we provide conclusions from our work. Further detailed modeling to understand our observational results in terms of asteroseismology will be presented in a separate paper.

\section{Observations}

\subsection{Photometry}

We obtained photometry at MDM and Baker observatories to support our spectroscopic observations, the details of which are provided in Table 1. Baker observatory (BO) is equipped with a $0.4 \mathrm{~m}$ telescope and a Roper Scientific RS1340b CCD photometer. At MDM we used the $1.3 \mathrm{~m} \mathrm{McGraw} \mathrm{Hill} \mathrm{telescope} \mathrm{with}$ an Apogee Instruments U47 CCD. For all of our CCD measurements, we binned the CCDs $2 \times 2$ pixels providing a dead time of $1 \mathrm{~s}$ and all used a red cut-off filter (BG38 or BG40), so the effective bandpass covers the $B$ and $V$ filters and is essentially that of a blue-sensitive photomultiplier tube. Accurate timing was accomplished via Network Time Protocol (NTP) connections to at least one stratum 1 and two stratum 2 servers. NTP timings were accurate to better than $0.02 \mathrm{~s}$.

Our photometric data were reduced using standard IRAF packages for image reduction, including bias subtraction, dark current and flat field corrections. Intensities were extracted using IRAF aperture photometry with extinction and cloud corrections using the normalized intensities of several field stars. As sdB stars are substantially hotter than typical field stars, differential light curves are not flat due to differential atmospheric and colour extinctions. A low-order polynomial was fit to remove these trends from the data on a night-by-night basis. Finally, the lightcurves are normalized by their average flux and centered around zero so the reported differential intensities are $\Delta I=(I /\langle I\rangle)-1$. Amplitudes are given as milli-modulation amplitudes (mma) with an amplitude of $10 \mathrm{mma}$ corresponding to $1.0 \%$ or 9.2 millimagnitudes. A portion of data from MDM is shown in Fig. 1. There is obvious beating that occurs on multiple time scales which indicates the multiperiodic nature of the pulsations.

\subsection{Spectroscopy}

During 7 nights in April 2006 we obtained nearly 5200 timeresolved low-resolution spectra of PG 1219, with the specifics of each run provided in Table 2. We obtained 2085 spectra at the Kitt Peak National Observatory's (KPNO) 4 m Mayall telescope using the RC spectrograph in long-slit mode. Our KPNO instrumental setup was: grating KPC-007 with the T2KB CCD which sampled approximately 3000-5000 A, a FWHM spectral resolution of about $4 \AA$, and a dispersion of $1.44 \AA /$ pixel. We subframed the CCD along the slit, reading out a section $2080 \times 37$ including a 41 column overscan region. Integration times were 


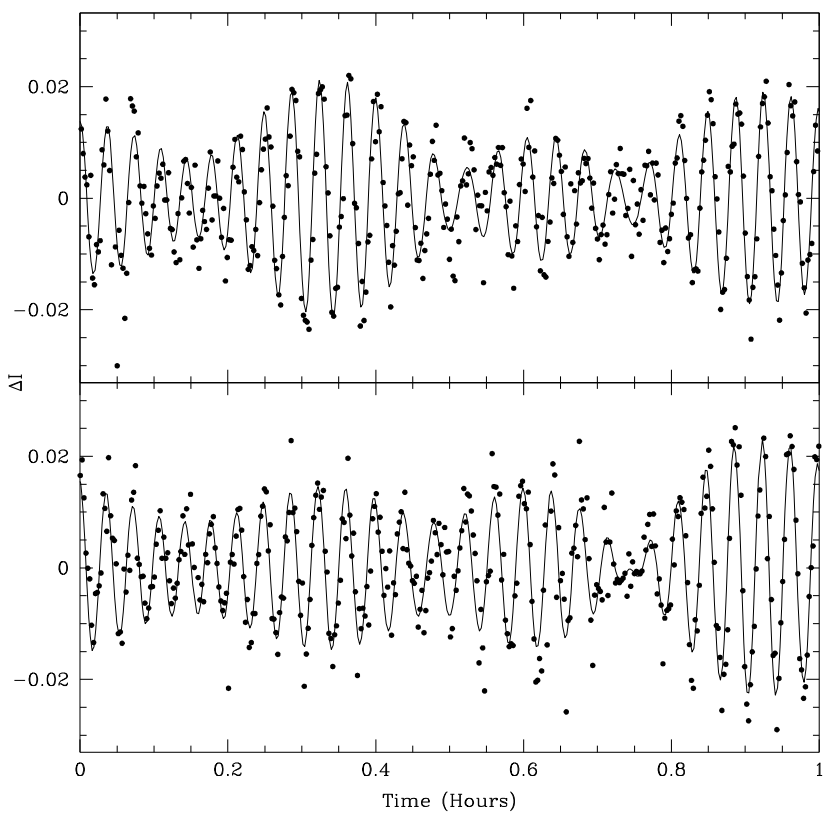

Fig. 1. Photometric lightcurves for consecutive $1 \mathrm{~h}$ segments of PG 1219. Solid line is a four-frequency fit to the points.

Table 2. Spectroscopic observations of PG 1219.

\begin{tabular}{lcccc}
\hline \hline $\begin{array}{l}\text { Date } \\
\text { 2006 }\end{array}$ & $\begin{array}{c}\text { Start time } \\
\text { (UT) }\end{array}$ & $\begin{array}{c}\text { Length } \\
\text { (h) }\end{array}$ & $\begin{array}{c}\text { Number of } \\
\text { spectra }\end{array}$ & Observat. \\
\hline 8 Apr. & $3: 23$ & 8.9 & 810 & KPNO \\
9 Apr. & $2: 47$ & 9.5 & 850 & KPNO \\
11 Apr. & $7: 34$ & 4.7 & 425 & KPNO \\
17 Apr. & $20: 43$ & 8.3 & 757 & NOT \\
18 Apr. & $20: 32$ & 8.9 & 760 & NOT \\
19 Apr. & $20: 29$ & 8.7 & 826 & NOT \\
20 Apr. & $20: 31$ & 8.0 & 756 & NOT \\
\hline
\end{tabular}

$10 \mathrm{~s}$ with rather long dead-times of $25 \mathrm{~s}$ for a total cycle time of $\sim 35 \mathrm{~s}$. Binning the CCD provided only negligible improvement, so we did not. We obtained helium-neon-argon arc-line spectra every 80-150 spectra aperiodically and observed the fluxstandard star Feige 66 three times per night. Halogen lamp flat fields were obtained nightly for removing pixel-to-pixel variations on the CCD.

We obtained 3099 spectra at the Nordic Optical Telescope (NOT) using ALFOSC in long-slit mode. Our NOT setup used grism \#16 and CCD \#8, which sampled approximately 3500-5050 А, a resolution of about $3 \AA$ FWHM at a dispersion of $0.77 \AA /$ pixel. The grism and slit were set up such that they were aligned with the rows of the CCD to shorten the dead times. Using $25 \mathrm{~s}$ integrations, we achieved a cycle time of $\sim 31 \mathrm{~s}$, so while the NOT integrated longer than KPNO, the cycle time was similar. A few re-acquisitions were done per night to correct the slit angle with the parallactic angle. Thorium-argon and helium arc-line spectra were obtained every 60-80 spectra. Halogen lamp images were obtained for flat-fielding.

The spectra were bias and dark-current corrected using overscan regions and flat field corrected using standard tasks within IRAF. Two bad columns in the NOT data were corrected by linear interpolation of pixels in adjacent columns. As the spectra contains low signal-to-noise $(\mathrm{S} / \mathrm{N})$ regions in the $\mathrm{UV}$, and the KPNO CCD had a sharp sensitivity decline redward of $5030 \AA$,

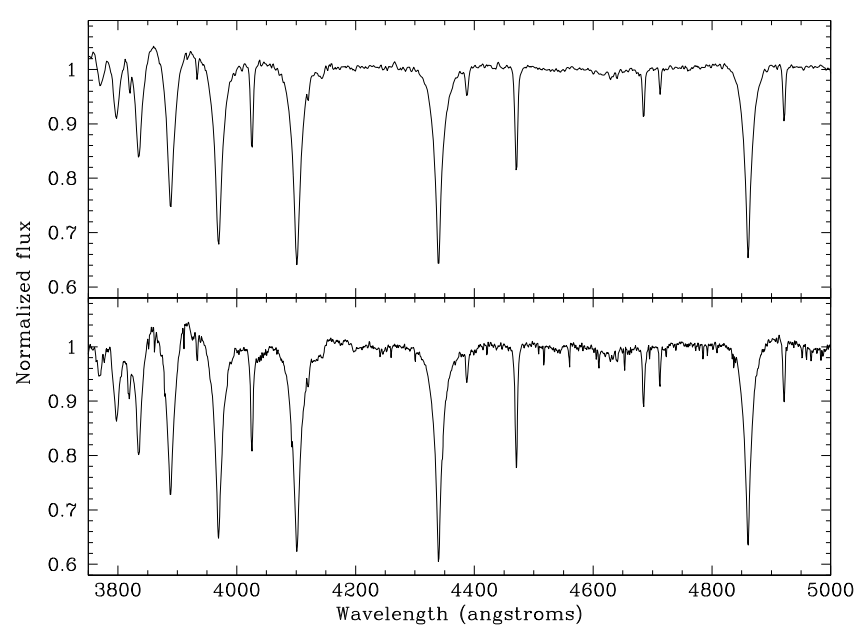

Fig. 2. Average of all normalized KPNO (top) and NOT (bottom) spectra.

the spectra were trimmed to $3710-5130 \AA$ for the KPNO data and 3480-5010 $\AA$ for the NOT data. One-dimensional spectra were optimally extracted after subtracting a fit to the sky background for each detector column. Wavelength calibration was done using the HeNeAr and the ThAr calibration spectra for the KPNO and NOT data, respectively, interpolating the wavelength solution between the nearest before and after calibration spectra. The average $\mathrm{S} / \mathrm{N}$ of the spectra was 31 for KPNO and 25 for the NOT data, so while the KPNO telescope was much larger than the NOT, the longer NOT integrations, combined with a more efficient grating allowed similar $\mathrm{S} / \mathrm{N}$ but higher spectral resolution in the NOT data. The spectra were normalized to the continuum using the IRAF task CONTINUUM which used a fourteenth order Legendre polynomial. The mean normalized KPNO and NOT spectra are shown in Fig. 2 on the same wavelength scale. The 10 and $25 \mathrm{~s}$ integration times lead to phase smearing that will reduce amplitudes of variation by 0.85 and $5.2 \%$, respectively, for a $140 \mathrm{~s}$ pulsation period. Additionally, our measurements are for disk-averaged observations and intrinsic amplitudes may be higher locally within the unresolved surface.

\section{The frequency content of the pulsations}

Our selection of PG 1219 as a target is partially due to its year-toyear stability and this continued during our 2006 observations. Table 3 provides the frequencies and amplitudes of pulsations detected during our four-year photometric program and indicates that two additional frequencies were observed during 2004, but during single nights only. However, during our 2006 campaign, a new, low amplitude frequency persisted through both weeks of observations. These occasionally-observed frequencies are labeled as $f 5$ through $f 7$ of Table 3. Pulsation spectra of photometry which overlaps our spectroscopic data are shown in Fig. 3. Insets show the spectral window (FT of a single sine-wave sampled at the same times as the data) and smaller panels show the residuals after prewhitening by the four main frequencies. The solid horizontal (blue) line is the $4 \sigma$ detection calculated as four times the average value of the FT for regions outside of areas of pulsation (Breger et al. 1994).

Photometric amplitudes of $f 1$ through $f 4$ show $20-40 \%$ variation over the course of four years, but during our spectroscopic observations, the amplitudes remain essentially constant. 
Table 3. Frequencies and amplitudes detected in our annual photometric data sets. All frequencies are given in $\mu \mathrm{Hz}$ and amplitudes in mma with least-squares errors on the last digits in parentheses.

\begin{tabular}{|c|c|c|c|c|c|c|c|c|}
\hline \multirow[t]{2}{*}{$\overline{\mathrm{ID}}$} & \multicolumn{2}{|l|}{$\overline{\overline{2003}}$} & \multicolumn{2}{|l|}{$\overline{2004}$} & \multicolumn{2}{|l|}{$\overline{2005}$} & \multicolumn{2}{|c|}{$\overline{\overline{2006}}$} \\
\hline & Freq. & Amp. & Freq. & Amp. & Freq. & Amp. & Freq. & Amp. \\
\hline$f 1$ & $6721.484(10)$ & $2.8(1)$ & $6721.529(55)$ & $3.9(2)$ & $6721.481(158)$ & $2.2(4)$ & $6721.591(4)$ & $2.9(1)$ \\
\hline$f 2$ & $6961.363(6)$ & $5.3(1)$ & $6961.355(33)$ & $6.6(2)$ & $6961.202(52)$ & $6.8(4)$ & $6961.489(2)$ & $6.4(1)$ \\
\hline$f 3$ & $7490.021(9)$ & $3.6(1)$ & $7489.727(38)$ & $5.7(2)$ & $7492.850(52)$ & $6.8(4)$ & $7490.833(2)$ & $4.5(1)$ \\
\hline$f 4$ & $7807.738(8)$ & 4.0(1) & $7807.771(29)$ & $7.4(2)$ & $7807.733(36)$ & $9.7(4)$ & $7807.857(2)$ & $6.1(1)$ \\
\hline$f 5$ & & & $7398.56(30)$ & $0.7(2)$ & & & & \\
\hline$f 6$ & & & & & & & $7744.6(45)$ & $1.5(1)$ \\
\hline$f 7$ & & & 8168.83(17) & $1.2(2)$ & & & & \\
\hline
\end{tabular}

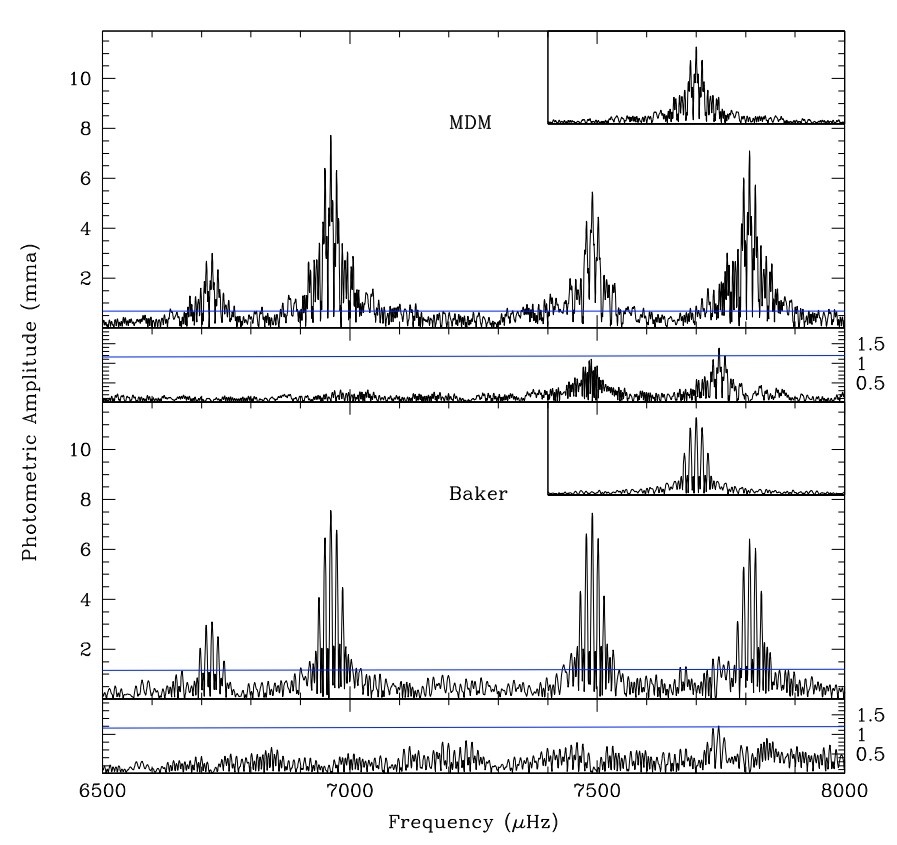

Fig. 3. Pulsation spectra of photometric data simultaneous with the spectroscopic observations. Smaller panels have the four main frequencies removed and insets are the spectral windows plotted on the same frequency scale. The (blue) lines indicate the $4 \sigma$ detection limit.

The only significant change is $f 3$, for which the amplitude increases by $14 \%$ in the week between the MDM and BO observations. From our photometric monitoring of PG 1219 we can conclude that there are four consistently detected frequencies and a fifth, low amplitude frequency.

Since our cycle time and S/N were better for photometry, we could examine the photometry for nightly variations in phase (defined as time of first maximum since JD $=2453830.0$ divided by the pulsation period). Such variations can cause integrated data to indicate lower amplitudes or have multiple peaks which are not intrinsic to the star. The phases of the four main frequencies were consistent to within the errors of those provided in Table 4, and so we conclude that no phase variations occurred during the photometric or spectroscopic runs. However the phases do change between the MDM and BO observations. The phase of $f 3$ changes by $13 \%$ while the others show marginal changes of 2,4 , and $3 \%$ for $f 1, f 2$, and $f 4$, respectively.

\subsection{Time-series spectroscopy}

The procedures we used are similar to those described in Telting \& Østensen (2004) and summarized as follows: we calculated

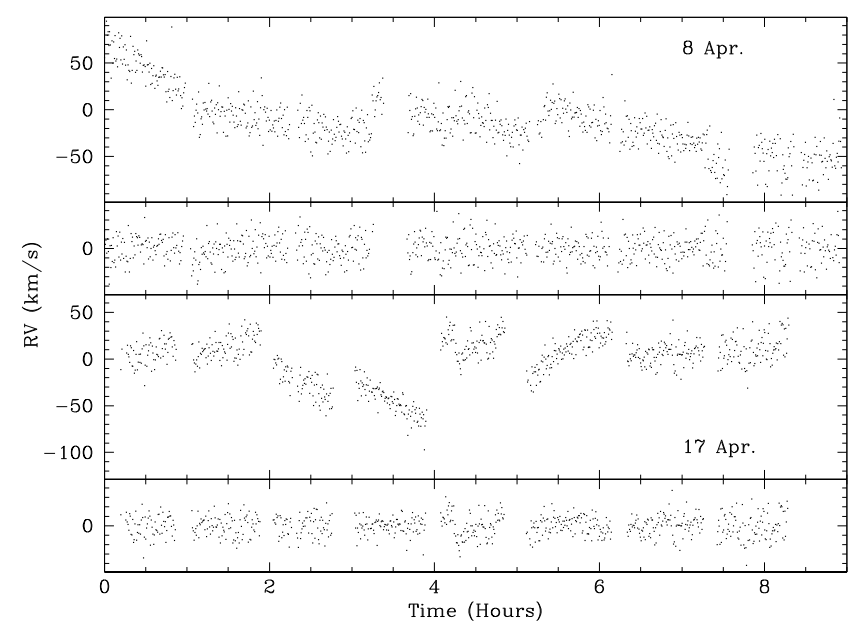

Fig. 4. Radial velocities obtained from cross-correlation of the time-resolved spectra. The top two panels show a run from KPNO both before and after fitting with polynomial segments and the bottom two panels show a run from the NOT before and after being fitted with polynomial segments. All panels are plotted to the same relative scale.

the radial velocity (RV) of the time-resolved spectra using the cross-correlation application FXCOR in IRAF. We produced nightly template spectra from the mean of individual spectra which FXCOR uses to do a Fourier cross-correlation between the template and individual spectra. We filtered the input to fit line features rather than large-scale trends in each spectrum, and a Gaussian was fit to the resulting cross-correlation function (CCF) of a best-fit size between 3 and 21 velocity bins around the maximum. We fit the $\mathrm{CCF}$ for the $\mathrm{H} \beta$ through $\mathrm{H} 8$ Balmer lines and the 4026 and $4471 \AA$ HeI lines. The radial velocity shifts are dominated by those of the strongest Balmer lines in the spectra. Figure 4 shows radial velocities obtained from one night each of KPNO and NOT data. The raw velocities, shown in the top portion for each date, show jumps in velocity due to repositioning the target on the slit and longer-term instrumental effects. These were corrected by fitting first or second-order polynomials to the segments between repositionings and are shown on the bottom portion for each date. All panels are plotted to the same scale but with varying velocity offsets. As these changes were of significantly longer time then the pulsations, there was no impact on pulsation velocities.

Figure 5 shows the temporal spectra of the KPNO and NOT RV data separately. The solid (blue) line shows the $4 \sigma$ detection limit and the insets show the spectral window. Results from least-squares fitting for the amplitudes and phases are provided in Table 4 in Sect. 4. Apparent in the figure is the missing peak 


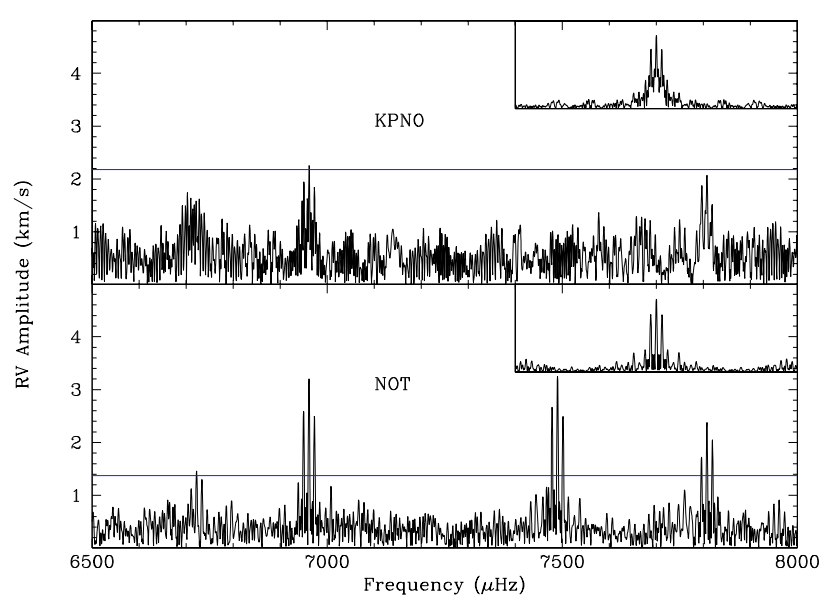

Fig. 5. Pulsation spectra of radial velocity data. Insets are the spectral windows and (blue) lines indicate the $4 \sigma$ detection limit.

where $f 3$ should be in the KPNO data. While the $4 \sigma$ limit is nearly double that of the NOT data, $f 2, f 3$, and $f 4$ have amplitudes in the NOT data which are sufficient to be detected in the KPNO data. $f 2$ and $f 4$ are both at least marginally detected, yet there is no trace of $f 3$ at all. $f 1$ shows a noisy "bump" in the appropriate place, but at just over $3 \sigma$, it is below the detection limit.

We also compute the equivalent widths $(E W)$ of the $\mathrm{H} \beta$ through $\mathrm{H} 8$ Balmer lines. For our $E W$ measurements, we shifted the spectra using the cross-correlation velocities to account for instrumental and pulsational velocity shifts. The resulting standard deviation in velocity is $0.07 \mathrm{~km} \mathrm{~s}^{-1}$ with an average CCF fit error of $5.34 \mathrm{~km} \mathrm{~s}^{-1}$. To reduce the noise in the wings of the profiles, each point in the profile was weighted with its own depth with respect to the continuum, using the following non-standard form, $E W=\sqrt{\Sigma(1-P(\lambda))^{2}} \times \Delta \lambda$. No long-term trends were evident in the $E W$ data, so no polynomial corrections were made. The results are shown in Fig. 6. $f 1$ is below the detection limit for both KPNO and NOT data, $f 2$ and $f 4$ are easily recovered in both sets, and $f 3$ is clearly detected in the NOT data, but just below $4 \sigma$ in the KPNO data. As evident in Fig. 6, NOT $E W$ values were found to be systematically larger than for the KPNO data. Analysis using various resolutions and line-bin-widths has shown the amplitudes to be dependent on both the resolution and the number of pixels used to determine the line widths. Because of these dependencies it is not feasible to compare the amplitudes between the two runs without some normalization. As the photometric and RV amplitudes of $f 4$ are the closest between the runs, in Table 4 we have normalized the KPNO $E W$ amplitudes to that of $f 4$ from the NOT run, while we show their original amplitudes in Fig. 6.

\section{Discussion}

\subsection{Effective temperature and gravity variations}

To measure changes in $T_{\text {eff }}$ and $\log g$ for individual pulsation frequencies, we folded the spectra into 20 phase bins according to the pulsation periods. By folding over each pulsation period, we average out the variations caused by the other three periods, effectively isolating those of the folded period. The phasebinned spectra have $\mathrm{S} / \mathrm{N}$ in the range 125 to 160 . We then fit these binned spectra with the LTE synthesis model atmospheres of Heber et al. (2000). The fitting is a $\chi^{2}$ process described by Bergeron et al. (1992) and updated to include helium abundance fits by Saffer et al. (1994). These model fits provide values of $T_{\text {eff }}, \log g$, and helium abundance as a function of pulsation phase for each of the periods. As the helium abundance did not change during any of the pulsations, we fixed this to the mean value and refit the models, mildly decreasing the fit errors on $T_{\text {eff }}$ and $\log g$. In Fig. 7 we illustrate the fitting procedure by showing the atmospheric fit to the template spectrum produced by combining all of the NOT spectra. The lines used for the fit are shown in the figure and include several Balmer lines and three He I lines. A single He II line is shown, but was not used in the fit. The fitted values for $T_{\text {eff }}, \log g$, and $N(\mathrm{He}) / N(\mathrm{H})$ are also provided on the figure and are nearly within the errors of those previously published (Charpinet et al. 2005; Heber et al. 2000; Koen et al. 1999; O'Toole \& Heber 2006). There are known systematic effects between LTE and NLTE model atmospheres with and without iron-group diffusion (Heber \& Edelmann 2004; O'Toole $\&$ Heber 2006) and so we do not claim any increased accuracy in our values, but merely show the lines to illustrate the fitting procedure. O'Toole \& Heber (2006) were able to overcome these inconsistencies using supersolar metal abundances, so we consider their measurements of $T_{\text {eff }}$ and $\log g$ as the most reliable. In our case we are not really concerned with the actual values of $T_{\text {eff }}$ and $\log g$ but the changes that occur through a pulsation cycle. In this sense, minor differences from previously reported values do not contribute to the fit errors in the variations.

\subsection{Fits of determined quantities}

To determine amplitudes and phases of the measurables (or model quantities), we used non-linear least-squares (NLLS) fits to measure the amplitudes and phases with the pulsation frequencies fixed to the those derived from photometry (which match those in velocity). For photometry, RV, and $E W$, we fit the data themselves with timing corrected to the barycenter of the solar system. For $T_{\text {eff }}$ and $\log g$, these are model fits to spectra folded over the frequency fixed to the photometric value. Then the variations in model $T_{\text {eff }}$ and $\log g$ were fit using our NLLS routine. The phase-folded data, along with the fits are shown in Figs. 8 through 11 with the quantities provided in Table 4. Frequency-folded data were produced from the photometric and $\mathrm{RV}$ data for the plot, but these were not fitted for the quantities in Table 4 except as noted ( $f 3 \mathrm{RV}$ and $f 1 \mathrm{EW}$ data for KPNO). Table 4 also provides the $4 \sigma$ detection limits from the data. No such values can be determined from the atmospheric model fits, though their formal errors have been folded into the NLLS formal errors shown in the figures and provided in the table.

Since we know that the pulsations are phase-stable during our photometric observations, we can expect them to be phasestable for the other measurables, and our results indicate it to be the case. Even the $13 \%$ change in phase for $f 3$ between the $\mathrm{MDM}$ and $\mathrm{BO}$ runs is measured, to within the errors for $\mathrm{RV}, E W$, $T_{\text {eff }}$, and $\log g$ between the KPNO and NOT runs, even though some amplitudes show large variations. This indicates that our phases are measured reliably.

\subsection{Amplitude ratios and phase differencing}

While we leave any attempts at mode identification to the second paper, which will properly simulate our results using pulsation models, it is still interesting to examine what we detected in such 
Table 4. Results of photometric and spectroscopic variations in PG 1219.

\begin{tabular}{lcccccc}
\hline \hline Measure & Obs. & $f 1$ & $f 2$ & $f 3$ & $f 4$ & Limit \\
\hline \multirow{5}{*}{ Photometric } & MDM & $2.9(2)$ & $7.9(2)$ & $5.6(2)$ & $7.2(2)$ & 0.6 \\
$(\mathrm{mma})$ & BO & $3.3(2)$ & $7.8(2)$ & $8.0(2)$ & $7.1(2)$ & 1.1 \\
$\mathrm{RV}$ & KPNO & $1.6^{a}(5)$ & $2.3(5)$ & $0.8^{b}(0.5)$ & $2.2(5)$ & 2.2 \\
$\left(\mathrm{~km} \mathrm{~s}^{-1}\right)$ & NOT & $1.4(3)$ & $3.1(3)$ & $3.0(3)$ & $2.4(3)$ & 1.2 \\
$E W\left(10^{-3}\right)$ & KPNO & $13.3^{b}(5.4)$ & $40.0(5.4)$ & $26.2^{a}(5.4)$ & $39.4(5.4)$ & 28.2 \\
& NOT & $16.2^{a}(3.8)$ & $34.7(3.8)$ & $34.8(3.8)$ & $39.4(3.8)$ & 18.2 \\
$T_{\text {eff }}$ & KPNO & $46(26)$ & $175(44)$ & $58(30)$ & $159(44)$ & \\
$(\mathrm{K})$ & NOT & $59(26)$ & $122(34)$ & $130(34)$ & $111(35)$ & \\
$\log g$ & KPNO & $12(7)$ & $24(7)$ & $15(8)$ & $17(6)$ & \\
$\left(10^{-3} \mathrm{~cm} \mathrm{~s}^{-2}\right)$ & NOT & $11(5)$ & $11(5)$ & $11(5)$ & $16(5)$ & \\
Photometric & MDM & $0.325(10)$ & $0.798(3)$ & $0.556(5)$ & $0.382(4)$ & \\
& BO & $0.310(11)$ & $0.838(5)$ & $0.423(4)$ & $0.354(5)$ & \\
RV & KPNO & $0.023^{a}(44)$ & $0.484(32)$ & $0.276^{a}(90)$ & $0.117(33)$ & \\
& NOT & $0.060(33)$ & $0.545(15)$ & $0.142(15)$ & $0.066(19)$ & \\
$E W$ & KPNO & $0.837^{a}(65)$ & $0.310(22)$ & $0.074(33)$ & $0.860(22)$ & \\
& NOT & $0.866^{a}(37)$ & $0.323(17)$ & $0.926(17)$ & $0.832(15)$ & \\
$T_{\text {eff }}$ & KPNO & $0.207(117)$ & $0.820(40)$ & $0.605(82)$ & $0.381(41)$ & \\
$\log g$ & NOT & $0.316(58)$ & $0.850(44)$ & $0.441(41)$ & $0.349(52)$ & \\
& KPNO & $0.392(113)$ & $0.794(51)$ & $0.444(72)$ & $0.384(69)$ & \\
& NOT & $0.182(74)$ & $0.802(63)$ & $0.417(61)$ & $0.350(52)$ & \\
\hline
\end{tabular}

Notes: ${ }^{a}$ indicates quantities were fit directly from the data, though they were below the $4 \sigma$ limit. ${ }^{b}$ indicates quantities that were fit from folded data (but not modeled). The KPNO $E W$ amplitudes have been normalized to that of $f 4$ of the NOT data.

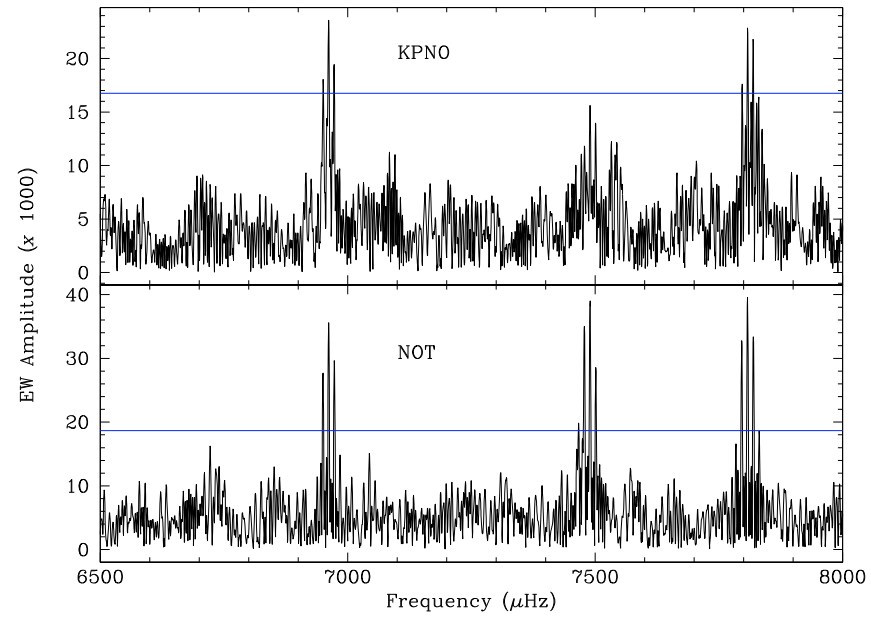

Fig. 6. Temporal spectra of equivalent width data.

a context. It is expected that pulsations of various modes will behave differently between photometry, velocity, equivalent width, effective temperature, and gravity measurements, and that such differences (and similarities) will provide strong constraints on mode identifications. There has already been a model for this star matched to photometric data (Charpinet et al. 2005) which concluded that our four measured frequencies should be associated with three different pulsation degrees. As such, we should expect observable differences between our measured quantities. The mode identifications of Charpinet et al. (2005) are provided in Fig. 12.

Figures 12 through 14 show amplitude ratios and phase differences of the observables using most of the available combinations. Figure 12 shows those quantities directly obtained from the data, Fig. 13 excludes photometry, but includes spectroscopic

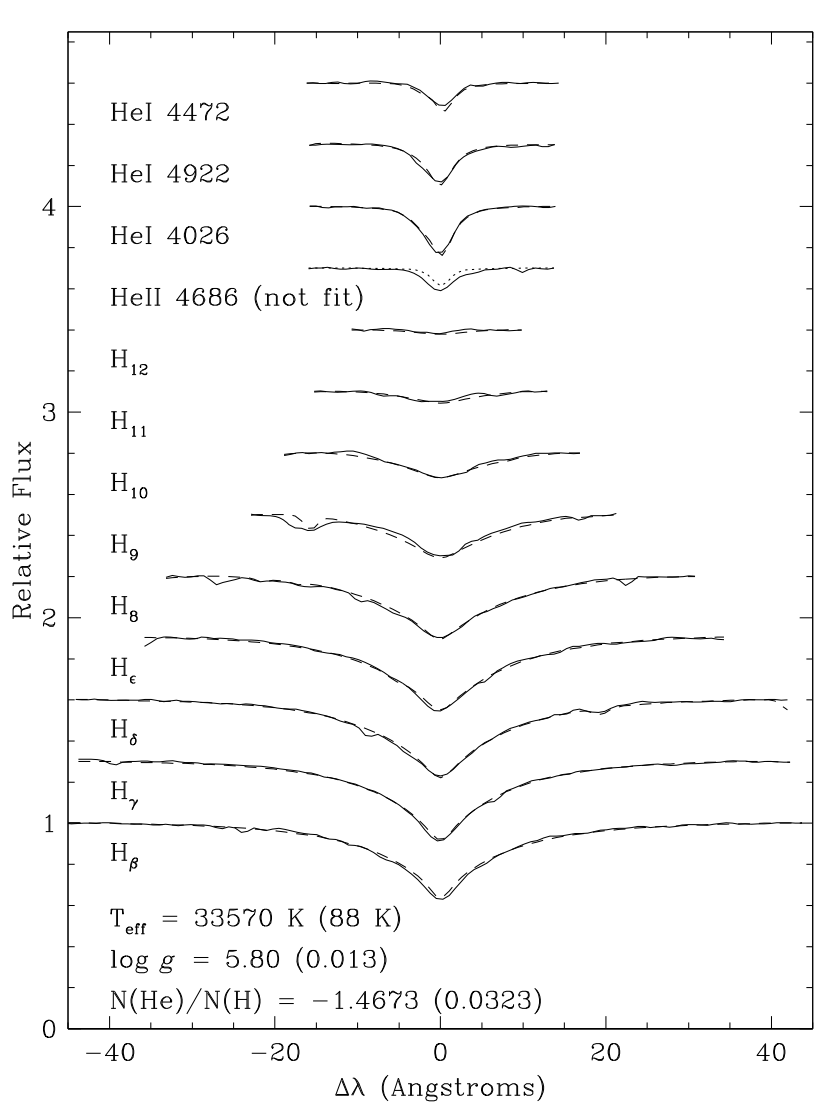

Fig. 7. Atmospheric models (dashed lines) fitted to the template spectrum of combined NOT data for several $\mathrm{H}$ and He I lines. The He II line is shown, but not used in the fit. Line identifiers and resultant atmospheric parameters are provided at the bottom. 


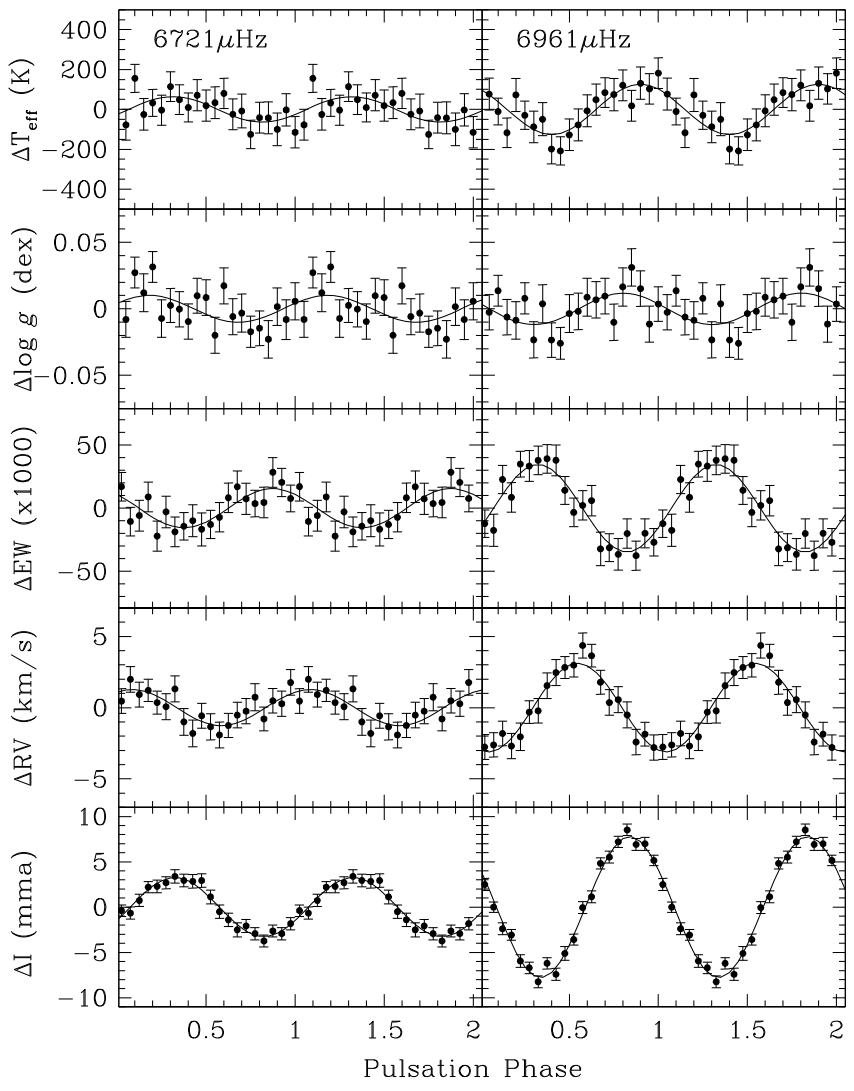

Fig. 8. Variations in $T_{\text {eff }}, \log g$, equivalent width, radial velocity, and photometry for $f 1=6721$ and $f 2=6961 \mu \mathrm{Hz}$ of the NOT/BO data, along with non-linear least-squares fits (solid lines). Phases shown twice for clarity.

quantities derived from models, and Fig. 14 compares the photometry with the model quantities. Values for the NOT/BO data have solid errorbars while those for the KPNO/MDM data have dotted errorbars. We note that most of our quantities are within the $1 \sigma$ errors of all other quantities, when the largely instrumental $E W$ offset between data sets is accounted for. While we do not attempt any mode identifications here, the similarities exhibited between the amplitude ratios suggest low degree modes with $\ell \leq 2$, which can behave similarly at most inclinations. The $\ell=3$ identification for $f 3$ seems unlikely since this frequency behaves very much like the others. However, there are no published model amplitude ratios by which to judge our results.

There are some expected relationships in phases that can be used to deduce the accuracy of the data as well. It is known that in low-degree modes, RV phases should be separated by one quarter $(\pi / 2)$ from $T_{\text {eff }}, \log g, E W$ and photometric phases for adiabatic pulsations. This has been observed for other sdBV stars (Telting \& Østensen 2004; Tillich et al. 2007), and we see the same thing. It should also be expected that brightness, $T_{\text {eff }}$, and $\log g$ should all be in phase but $E W$ should be in anti-phase to these observables, and again, this is what we observe. Expected phase relations, deduced from atmospheric models are indicated as dashed lines in the figures. Our observations scatter tightly around these lines, indicating that our spectroscopic reductions and model fitting are reliable.

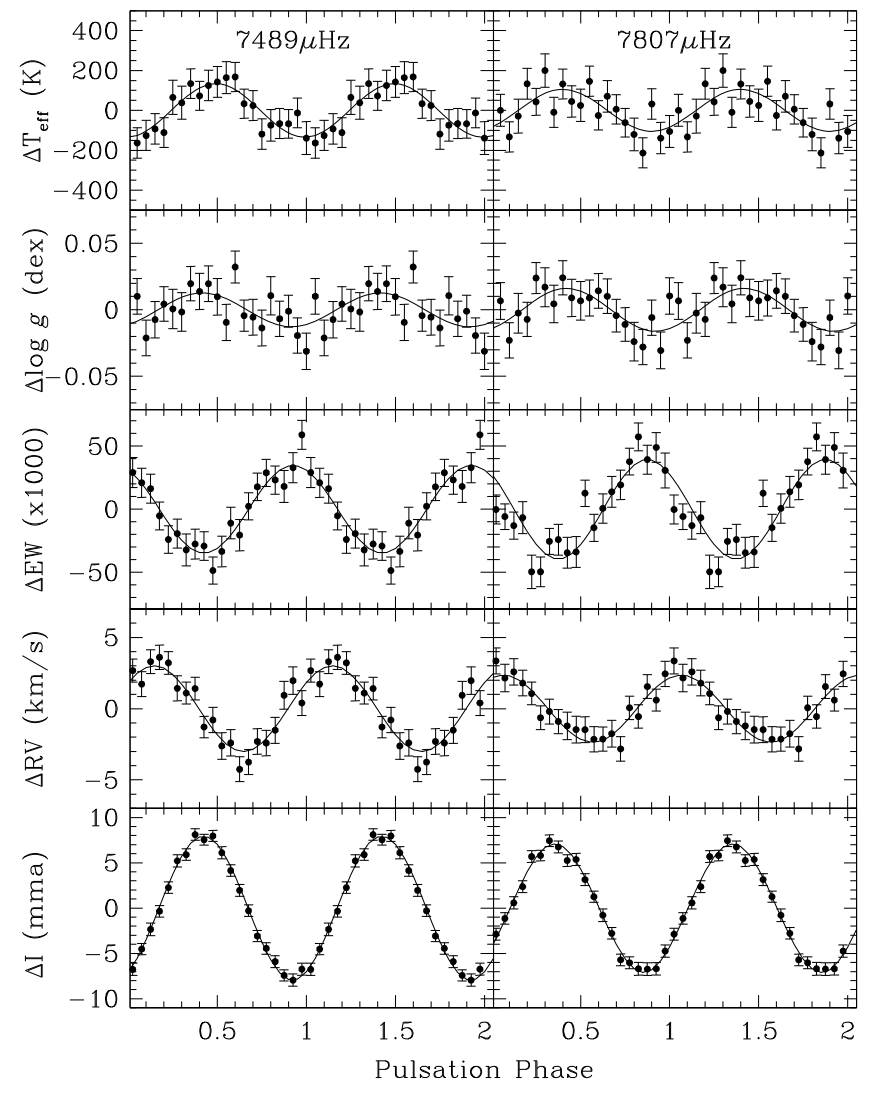

Fig. 9. Same as Fig. 8 for $f 3=7489$ and $f 4=7808 \mu \mathrm{Hz}$ of the NOT/BO data.

\section{Conclusions}

During 2006 we collected simultaneous photometry and timeseries spectroscopy from MDM and Kitt Peak Observatories and Baker Observatory and the NOT of the pulsating subdwarf B star PG 1219+534. In total, we obtained over 5000 spectra and nearly 15000 photometric measurements. We have recovered the four frequencies of the discovery paper (Koen et al. 1999) in photometric, velocity, and equivalent width variations.

From four years of photometry, we determine that PG 1219 has four consistent frequencies with occasional low-amplitude transients. One such transient occurred during our spectroscopic runs, though we did not recover that frequency in any of our spectroscopically-determined quantities. The photometric amplitudes of $f 1, f 2$, and $f 4$ remain stable over the course of our observations but the amplitude of $f 3$ increased by $43 \%$ between our MDM and BO observations. Similarly, the photometric phases were stable during our observations, but most varied between the runs. That of $f 3$ showed the largest change in phase of $13 \%$ while $f 2$ and $f 4$ had changes of 4 and $3 \%$, respectively. Only $f 1$ had no phase changes, to within the errors.

Our spectroscopic results were similar, in that only $f 3$ showed significant amplitude variations between the runs. It has a very low RV amplitude of $0.8 \mathrm{~km} \mathrm{~s}$ in the KPNO data, which grows to an amplitude of $3.0 \mathrm{~km} \mathrm{~s}^{-1}$ in the NOT data. However, the differences in normalized $E W$ amplitudes are within the errors for all frequencies.

By folding the spectra over the pulsation period, we were able to fit atmospheric models to the higher $\mathrm{S} / \mathrm{N}$ binned 


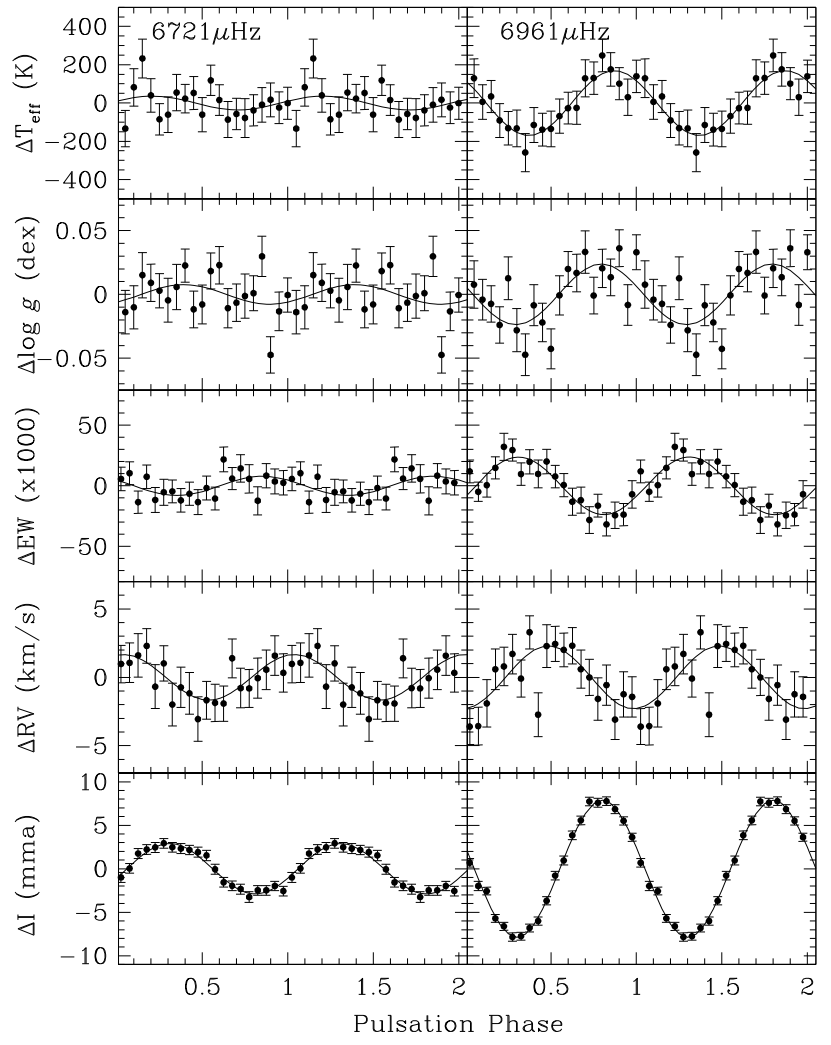

Fig. 10. Same as Fig. 8 for KPNO/MDM data.

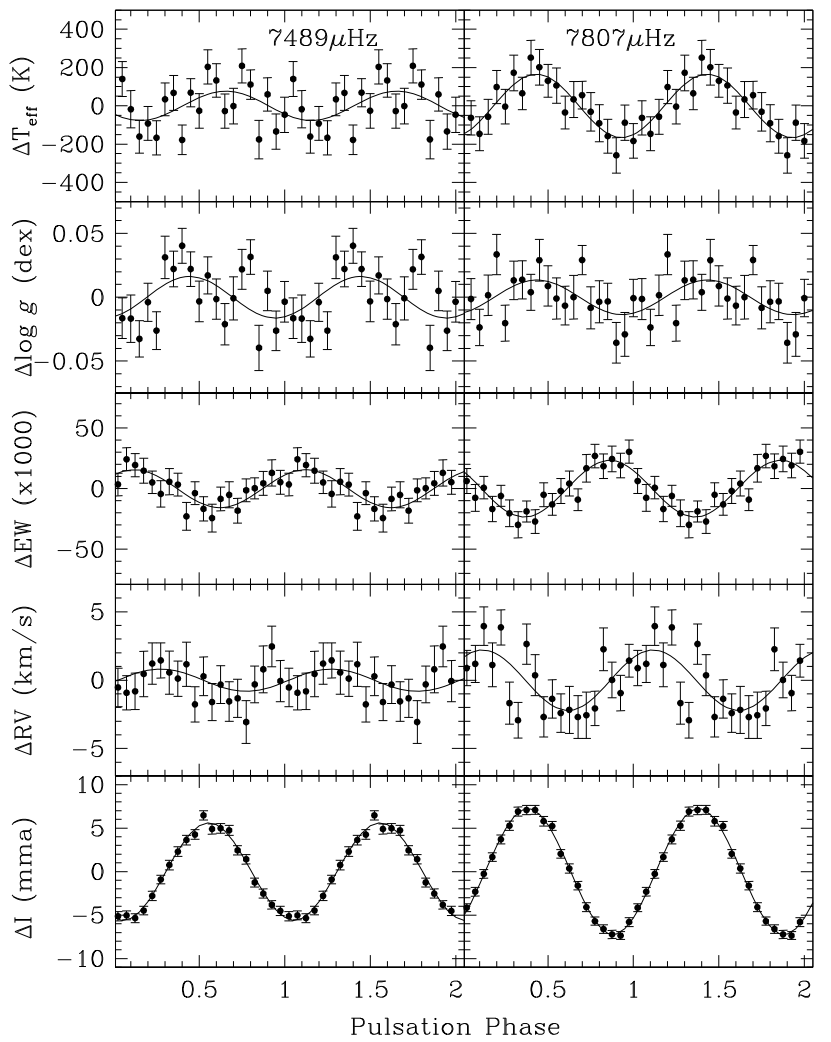

Fig. 11. Same as Fig. 9 for KPNO/MDM data.

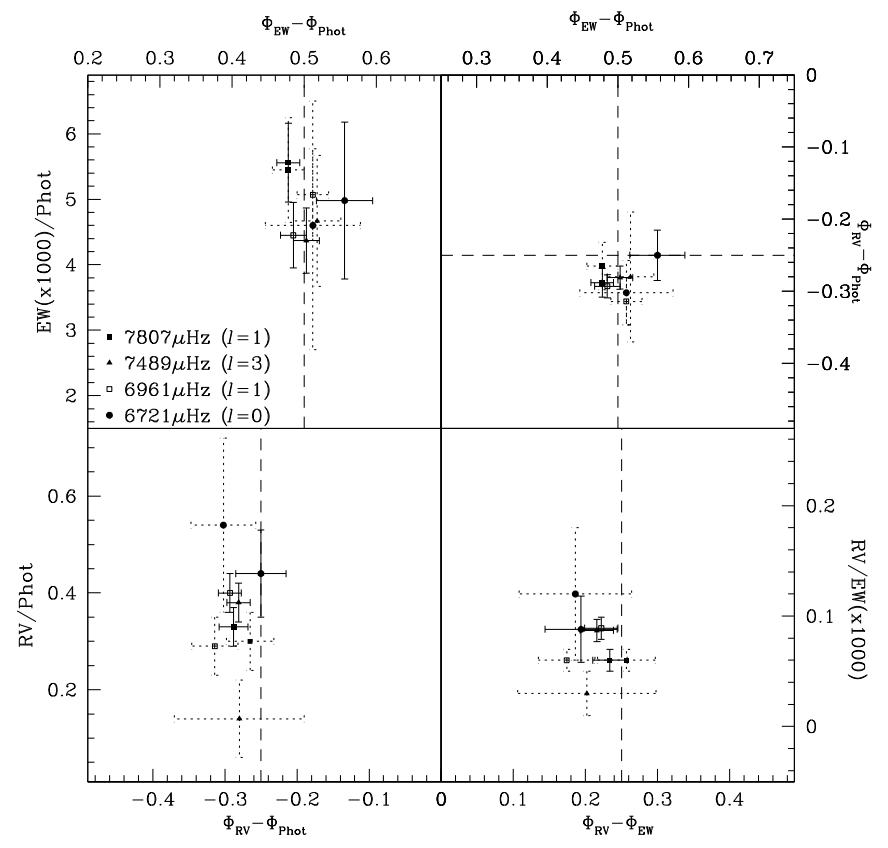

Fig. 12. Amplitude ratios versus phase differences for fitted observables. Solid errorbars indicate NOT/BO data while dotted errorbars indicate KPNO/MDM data. Dashed (blue) lines indicate expected phases from model simulations and mode identifications are those of Charpinet et al. (2005).

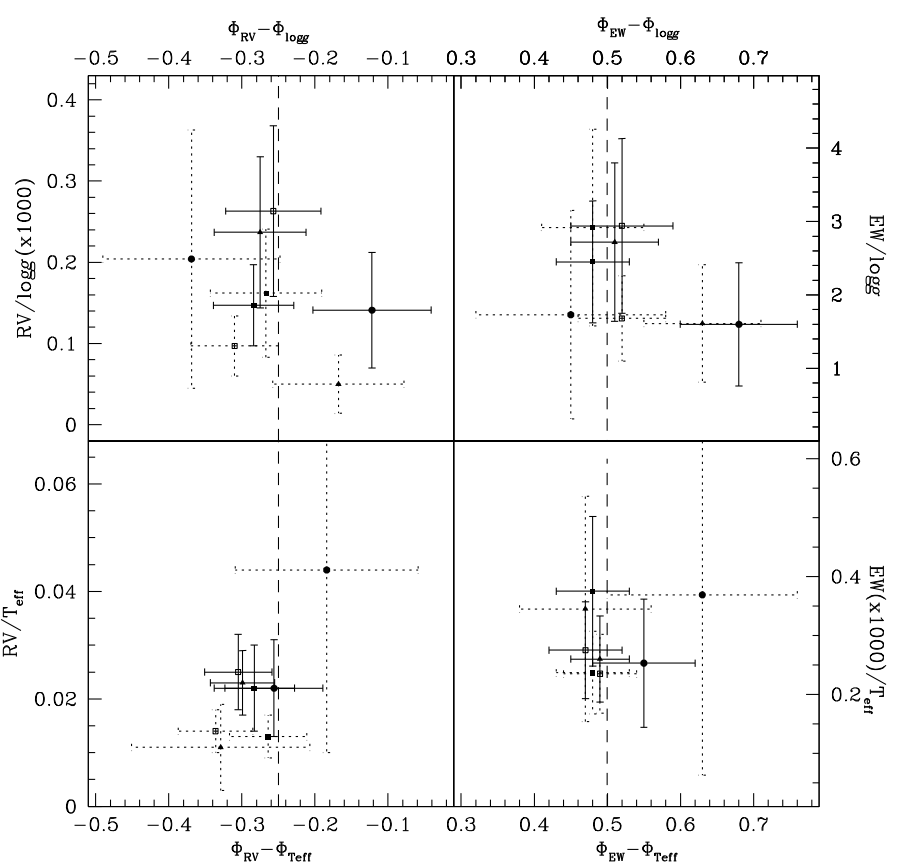

Fig. 13. Same as Fig. 12 for quantities determined solely from spectroscopy.

spectra to extract $T_{\text {eff }}$ and $\log g$ variations for all four frequencies. Again all pulsation amplitudes are consistent between the KPNO and NOT data, except for $f 3$ in $T_{\text {eff }}$ which more than doubles. As such, $f 3$ has significant amplitude increases of 43 , 275 , and $124 \%$ between the KPNO/MDM and NOT/BO observations for photometry, $\mathrm{RV}$, and $T_{\mathrm{eff}}$, respectively. While there is a $33 \%$ increase in $E W$ amplitude between the runs for $f 3$, it is just within the $1 \sigma$ errors. 


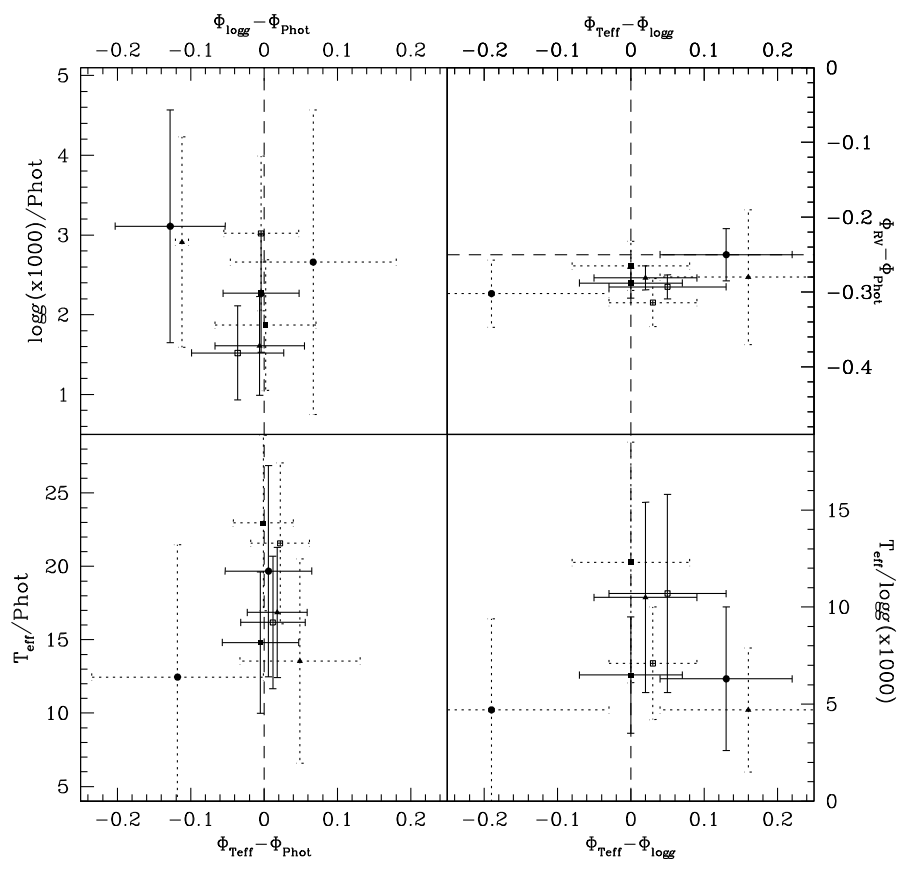

Fig. 14. Same as Fig. 12 comparing photometry and model-fitted quantities.

We examined amplitude ratios and phase differences for various measurables for each frequency. We will interpret these more fully in a subsequent paper, but observationally, we can conclude that the ratios and differences do not differ greatly between frequencies. The simplest interpretation of this result is that they are all low-degree $(\ell \leq 2)$ modes. We also compared phase differences to those expected from adiabatic atmospheric models. Our measured differences match those expected, except for small departures which are most likely caused by nonadiabatic effects.

A large arsenal of quantities can now be applied to identify the modes of pulsation. A future paper will match our measured quantities to those from perturbed synthetic pulsation spectra to constrain the modes of each frequency. This work is also the first successful application of time-series spectroscopy to a "normal" amplitude sdBV star. All previous detections were of stars with unusually high photometric amplitudes (O'Toole et al. 2000, 2002, 2003, 2005; Woolf et al. 2002; Telting \& Østensen 2004, 2006; Tillich et al. 2007) and so our work indicates that such studies can provide useful measurements for the majority of sdBV stars.

Acknowledgements. We would like to thank Simon Jeffery for some of the atmospheric models and fitting routines used in our analysis and the NOT, MDM, and KPNO TACs for time allocations. M.D.R. was supported by an American Astronomical Society Small Research Grant and the National Science Foundation Grant AST007480. Any opinions, findings, and conclusions or recommendations expressed in this material are those of the author(s) and do not necessarily reflect the views of the American Astronomical Society or the National Science Foundation. M.D.R. would also like to thank Conny Aerts and a HELAS travel grant which allowed us to congregate in Leuven to work on atmospheric models. J.R.E., S.L.H., and R.L.V.W. were supported by the Missouri Space Grant Consortium and an REU Supplement grant from the National Science Foundation. R. $\varnothing$. is supported by the Research Council of the University of Leuven and the FP6 Coordination Action HELAS of the EU. Some of the data presented here have been taken using ALFOSC, which is owned by the Instituo de Astrofisica de Andalucia (IAA) and operated at the Nordic Optical Telescope under agreement between IAA and the NBIfAFG of the Astronomical Observatory of Copenhagen. Based on observations made with the Nordic Optical Telescope, operated on the island of La Palma jointly by Denmark, Finland, Iceland, Norway, and Sweden, in the Spanish Observatorio del Roque de los Muchachos of the Instituto de Astrofisica de Canarias.

\section{References}

Bergeron, P., Saffer, R. A., \& Libert, J. 1992, ApJ, 394, 228

Breger, M., Ostermann, W. M., Shi-Yang, J., et al. 1994, A\&A, 289, 162

Charpinet, S., Fontaine, G., Brassard, P., Green, E. M., \& Chayer, P. 2005, A\&A, 437,575

D’Cruz, N. L., Dorman, B., Rood, R. T., \& O’Connell, R. W. 1996, ApJ, 466, 359

Fontaine G., Green, E. M., Chayer, P., Brassard, P., Charpinet, S., \& Randall, S. K. 2006, BaltA, 15, 211

Green, E. M., Fontaine, G., Reed, M. D., et al. 2003, ApJ, 583, L31

Han, Z., Podsiadlowski, Ph., Maxted, P. F. L., Marsh, T. R., \& Ivanova, N. 2002, MNRAS, 336, 449

Han, Z., Podsiadlowski, Ph., Maxted, P. F. L., \& Marsh, T. R. 2003, MNRAS, 341, 669

Harms, S. L., Reed, M. D., \& O’Toole, S. J. 2006, BaltA, 15, 251

Heber, U. 1984, A\&A, 130, 119

Heber, U., \& Edelmann, H. 2004, Ap\&SS, 291, 341

Heber, U., Reid, I. N., \& Werner, K. 2000, A\&A, 363, 198

Jeffery, C. S., \& Pollacco, D. 2000, MNRAS, 318, 974

Kazarovets, E. V., Kireeva, N. N., Samus, N. N., \& Durlevich, O. V. 2003, IBVS, 5422

Kilkenny, D., Koen, C., O’Donoghue, D., \& Stobie, R. S. 1997, MNRAS, 285, 640

Kilkenny D., Koen, C., O’Donoghue, D., et al. 1999, MNRAS, 303, 525

Koen C., O’Donoghue, D., Pollacco, D. L., \& Charpinet, S. 1999, MNRAS, 305, 28

Kuassivi, Bonanno, A., \& Ferlet, R. 2005, A\&A, 442, 1015

O'Toole, S. J., \& Heber, U. 2006, A\&A, 452, 579

O'Toole, S. J., Bedding, T. R., Kjeldsen, H., et al. 2000, ApJ, 537, L53

O’Toole, S. J., Bedding, T. R., Kjeldsen, H., Dall, T. H., \& Stello, D. 2002, MNRAS, 334, 471

O’Toole, S. J., Jørgensen, M. S., Kjeldsen, H., et al. 2003, MNRAS, 340, 856

O'Toole, S. J., Falter, S., Heber, U., et al. 2004, A\&A, 440, 667

Reed, M. D., Green, E. M., Callerame, K., et al. 2004a, ApJ, 607, 445

Reed, M. D., Kawaler, S. D., Zola, S., et al. (The Whole Earth Telescope Collaboration) 2004b, MNRAS, 348, 1164

Reed, M. D., Eggen, J. R., Zhou, A.-Y., et al. 2006, MNRAS, 369, 1529

Reed, M. D., Terndrup, D. M., Zhou, A.-Y., et al. 2007a, MNRAS, 378, 1049

Reed, M. D., O’Toole, S. J., Terndrup, D. M., et al. 2007b, ApJ, 664, 518

Saffer, R. A., Bergeron, P., Koester, D., \& Liebert, J. 1994, ApJ, 432, 351

Telting, J. H., \& Østensen, R. H. 2004, A\&A, 419, 685

Telting, J. H., \& Østensen, R. H. 2006, A\&A, 450, 1149

Tillich, A., Heber, U., O’Toole, S. J., Østensen, R., \& Schuh, S. 2007, A\&A, 473, 219

Woolf, V. M., Jeffery, C. S., \& Pollacco, D. L. 2002, MNRAS, 329, 497

Zhou, A.-Y., Reed, M. D., Harms, S., et al. 2006, MNRAS, 367, 179 